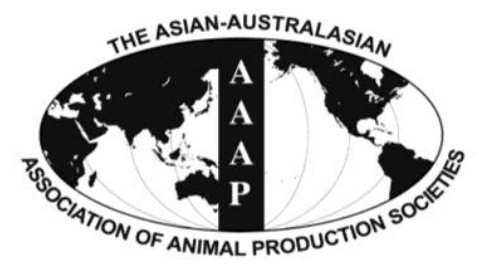

\title{
Transgenic Expression of MsHsp23 Confers Enhanced Tolerance to Abiotic Stresses in Tall Fescue
}

\author{
Ki-Won Lee, Gi Jun Choi, Ki-Yong Kim, Hee Jung Ji, Hyung Soo Park, Yong-Goo Kim, \\ Byung Hyun Lee ${ }^{1}$ and Sang-Hoon Lee* \\ Grassland and Forages Division, National Institute of Animal Science, \\ Rural Development Administration, Cheonan, 330-801, Korea
}

\begin{abstract}
Tall fescue (Festuca arundinacea Schreb.) is an important cool season forage plant that is not well suited to extreme heat, salts, or heavy metals. To develop transgenic tall fescue plants with enhanced tolerance to abiotic stress, we introduced an alfalfa Hsp23 gene expression vector construct through Agrobacterium-mediated transformation. Integration and expression of the transgene were confirmed by polymerase chain reaction, northern blot, and western blot analyses. Under normal growth conditions, there was no significant difference in the growth of the transgenic plants and the non-transgenic controls. However, when exposed to various stresses such as salt or arsenic, transgenic plants showed a significantly lower accumulation of hydrogen peroxide and thiobarbituric acid reactive substances than control plants. The reduced accumulation of thiobarbituric acid reactive substances indicates that the transgenic plants possessed a more efficient reactive oxygen species-scavenging system. We speculate that the high levels of $M s H s p 23$ proteins in the transgenic plants protect leaves from oxidative damage through chaperon and antioxidant activities. These results suggest that MsHsp23 confers abiotic stress tolerance in transgenic tall fescue and may be useful in developing stress tolerance in other crops. (Key Words: Tall Fescue, Forage, Agrobacterium tumefaciens, MsHsp23, Transformation)
\end{abstract}

\section{INTRODUCTION}

Plant small heat shock proteins (sHSPs) are nuclearencoded proteins that have been classified into 5 multigene families. Interestingly, sHSPs have been shown to localize to a number of different cellular compartments, including the cytosol, chloroplast, endoplasmic reticulum, and mitochondrium (Vierling, 1991). Recently, it has been recognized that sHSPs have protective roles against a variety of stresses in addition to high temperatures. These include induction of the expression of 20 to $40 \mathrm{kDa}$ HSPs by gamma-ray irradiation in tomatoes (Ferullo et al., 1994), ER-localized sHSP induction by cold storage in potato tubers, cytosolic sHSP induction by pathogenesis and ozone fumigation in parsley leaves (Eckey-Kaltenbach et al., 1997), and induction of mitochondrial and cytosolic sHSPs by oxidative stress in tomato suspension cultures (Banzet et

\footnotetext{
* Corresponding Author: S. -H. Lee. Tel: +82-41+580-6754, Fax: +82-41+580-6759, E-mail: sanghoon@korea.kr

${ }^{1}$ Division of Applied Life Science (BK21 Program), Gyeongsang National University, Jinju, 600-701, Korea.

Submitted Jan. 15, 2012; Accepted Mar. 7, 2012; Revised Mar. 29, 2012
}

al., 1998).

Tall fescue (Festuca arundinacea Schreb.) is a coolseason perennial grass grown for forage and turf. Tall fescue grows best in cool, moist environments and is not well suited to extreme heat, cold, or prolonged drought (Hannaway et al., 2005). Abiotic stress causes declines in forage and turf quality, which have been associated with reductions in root growth, leaf water potential, cell membrane stability, photosynthetic rate, and carbohydrate accumulation (Jiang and Huang, 2000). Most of the forage grasses for feedstuff grown in Korea were originally introduced from foreign countries. However, their harvest and quality is considerably lower when they are grown in Korea because of various environmental stresses under changed conditions. However, most of idle lands including unused farmlands yields are very poor because of adverse environmental conditions. Alfalfa mitochondrial small heatshock protein $(M s H s p 23)$ is a multifunctional protein that enhanced tolerance of environmental stresses (Lee et al., 2012a; Lee et al., 2012b). To the best of our knowledge, however, there have been no reports on the overexpression of $M s H s p 23$ in forage grasses. In this study, we 
overexpressed an alfalfa MsHsp23 cDNA clone in tall fescue plants in order to investigate the effect of the transgene on abiotic tolerance in tall fescue.

\section{MATERIALS AND METHODS}

\section{Gene construct and Agrobacterium-mediated transformation}

The MsHsp23 cDNA fragment was ligated into the translation initiation codon within the $5^{\prime}$ untranslated sequence of the constitutively expressed cauliflower mosaic virus promoter $(35 \mathrm{~S})$. The resultant chimeric gene cassette was then inserted into the KpnI/XbaI site of a pCAMBIA1300 binary vector (Figure 1). Recombinant pCAM-MsHsp23 was introduced into Agrobacterium tumefaciens strain EHA105, which was then used for genetic transformation. Callus induction and Agrobacterium-mediated genetic transformation were performed according to our previous protocol (Lee et al., 2004; Lee et al., 2007).

\section{Screening of transgenic plants}

Putative hygromycin-resistant transgenic plants were selected by PCR analysis. Genomic PCR was performed using the following primers: the MsHsp23 gene: 5'TCTAGAATGGCGTCTGTTGCT-3' and 5'-TTCTCAGCT ATTTAGGAGC-TC-3'; the $h p t$ gene: 5'-CCTGAACTC ACGACG-3' and 5'-AAGA-CCAAGGAGCATAT-3'.

\section{Expression analysis of transgenic plants}

RNA extraction and hybridization were performed as previously described (Lee et al., 2006). Total RNA was isolated using the Plant RNeasy mini kit (Qiagen, CA) following the manufacturer's instructions. After isolation, total RNA was treated with RNase-free DNase (Invitrogen, CA) to ensure the complete removal of genomic DNA. Gene-specific DNA probes were amplified by PCR using MsHsp23 as the template. Subsequently, ${ }^{32} \mathrm{P}$-labeled probes were generated by random primer synthesis incorporating ${ }^{32}$ P-dATP by using the Prime-it ${ }^{\circledR}$ II kit (Stratagene, USA).

Protein extraction and Western blot analysis were conducted as described previously (Kim et al., 2010). Briefly, total proteins were subjected to SDS-PAGE and then transferred to nitrocellulose membranes (Amersham Biosciences). The blotted membranes were blocked for $1 \mathrm{~h}$ in TTBs $(50 \mathrm{mM}$ Tris-HCl, $\mathrm{pH} 8.2,0.1 \%$ v/v Tween 20, and $150 \mathrm{mM} \mathrm{NaCl}$ ) containing $5 \% \mathrm{w} / \mathrm{v}$ nonfat dry milk and subsequently incubated with an anti-MsHsp23 rabbit antibody at a 1:10,000 dilution for $2 \mathrm{~h}$ followed by a secondary anti-rabbit IgG HRP $(\mathrm{H}+\mathrm{L})$ liquid antibody conjugated with peroxidase diluted $1: 1,000$ in TTBs. The immunoblot signals were detected using ECL (Perkin Elmer Life Sciences, USA) and visualized on X-ray films (Fuji Medical X-ray film, Japan).

\section{Plant growth and imposition of stresses}

Transgenic tall fescue plants were grown in soil pots in a growth chamber maintained at $25^{\circ} \mathrm{C}$ under a $16 / 8-\mathrm{h}$ photoperiod. Six weeks after transplantation, the plants were exposed to stress, and the samples were collected for physiological analyses as described earlier (Lee et al., 2007).

For salt or arsenic $\left(\mathrm{Na}_{2} \mathrm{HAsO}_{4} \cdot 7 \mathrm{H}_{2} \mathrm{O}\right)$ treatments, the pots were irrigated with solutions of $300 \mathrm{mM}$ salt or 500 $\mu \mathrm{M}$ arsenic for 2 wks. The control plants were irrigated with tap water. Leaf samples were harvested 1 and 2 wks after treatment and used for analysis.

\section{Determination of lipid peroxidation and $\mathrm{H}_{2} \mathrm{O}_{2}$ levels}

Measurements of lipid peroxidation and $\mathrm{H}_{2} \mathrm{O}_{2}$ concentrations were performed as described previously (Lee et al., 2007). The thiobarbituric acid reactive substance (TBARS) concentration in leaf samples from control and transgenic plants was determined according to Ekmekci and Terzioglu (2005), using an extinction coefficient of 155 $\mathrm{mM}^{-1} \mathrm{~cm}^{-1}$. The $\mathrm{H}_{2} \mathrm{O}_{2}$ concentration in leaf samples from control and transgenic plants was measured spectrophotometrically according to Lin and Kao (2001).

\section{RESULTS AND DISCUSSION}

\section{Integration and expression of transgenes in transgenic plants}

Sterilized mature seeds of the most widely cultivated tall fescue cultivar, Kentucky-31, were used as explants to induce callus formation. Agrobacterium-mediated genetic transformation was performed according to our previous

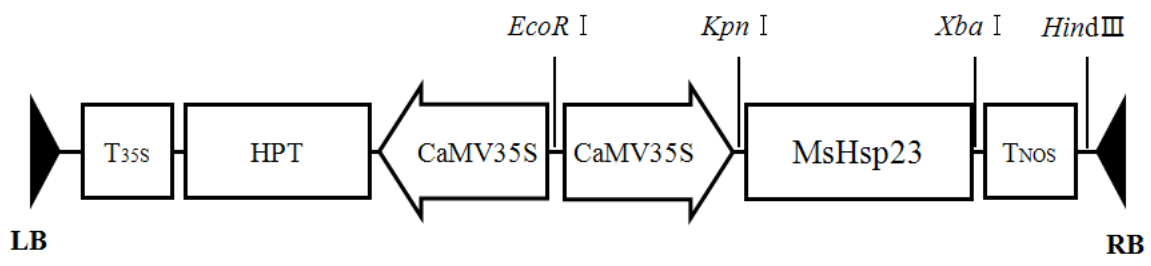

Figure 1. Schematic representation of the T-DNA region of pCAM- $M s H s p 23$, the expression vector used for transformation. RB, right border; LB, left border; 35S, CaMV 35S promoter; HPT, hygromycin phosphotransferase; MsHsp23, alfalfa small heat shock protein, $\mathrm{T}_{35 \mathrm{~S}}$, CaMV $35 \mathrm{~S}$ terminator; $\mathrm{T}_{\mathrm{NOS}}$, nopaline synthase terminator; EcoRI , KpnI, XbaI and HindIII restriction sites. 
protocol (Lee et al., 2004; Lee et al., 2007). Eight independent primary transformants recovered from hygromycin-containing selection medium showed the presence of the gene as determined by PCR screening using gene-specific primers for both the hpt and MsHsp23 genes (Figure 2).

For examining the expression of the transgenes, total RNA was isolated and analyzed by RNA blots (Figure 3). The RNA gel blots suggested that the CaMV 35S promoter was driving high-level expression of the MsHsp23 gene. By contrast, no transcripts were observed in the non-transgenic control plants. The expression of the transgene at the protein level was confirmed by immunoblotting. As shown in Figure 4, the antibody recognized a protein with a molecular mass of approximately $23 \mathrm{kDa}$ in the samples obtained from the transgenic plants, whereas no signal was detected in the control line. These results indicated that the transgene was successfully expressed transcriptionally and translationally in the transgenic tall fescue plants.

\section{Reduction of ROS accumulation and lipid peroxidation}

Stress-induced changes are frequently related to an increase in membrane permeability. Under stress conditions, increased membrane permeability can affect both membrane integrity and cell compartmentation (Leshem, 1992). Increased levels of reactive oxygen species (ROS) are generated under a variety of stresses, including salt and heavy metals (Cheeseman, 2007), and are reflected in the $\mathrm{H}_{2} \mathrm{O}_{2}$ levels in the tissue. Thus, we quantified $\mathrm{H}_{2} \mathrm{O}_{2}$ content in transgenic and control plants in order to assess cellular

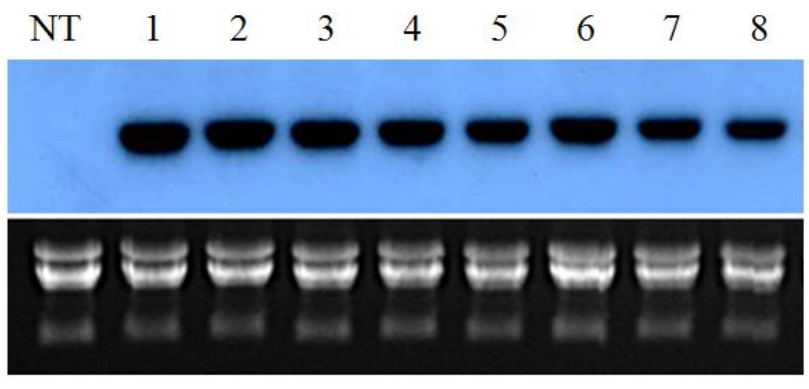

Figure 3. Northern blot analysis of the $M s H s p 23$ gene in control and transgenic tall fescue plants. Total RNA $(10 \mu \mathrm{g})$ was separated by electrophoresis on an agarose gel, blotted on a nylon membrane, and hybridized with ${ }^{32} \mathrm{P}$-labeled $\mathrm{MsHsp} 23$ specific cDNA probe.

conditions when MsHsp23 is over accumulated. Under control conditions, we did not detect any marked differences between control and transgenic plants. However, transgenic plants showed significantly lower $\mathrm{H}_{2} \mathrm{O}_{2}$ content in response to salt and arsenic treatments (Figure 5). These results suggest that the transgenic plants have a greater capability for maintaining membrane stability under stress conditions.

Malondialdehyde (measured as TBARS content) is one of the final products of stress-induced lipid peroxidation of polyunsaturated fatty acids. TBARS formation in plants exposed to adverse environmental conditions is a reliable indicator of cellular free-radical generation. In order to determine the level of membrane damage caused by salt and arsenic treatments, we examined lipid peroxidation in the

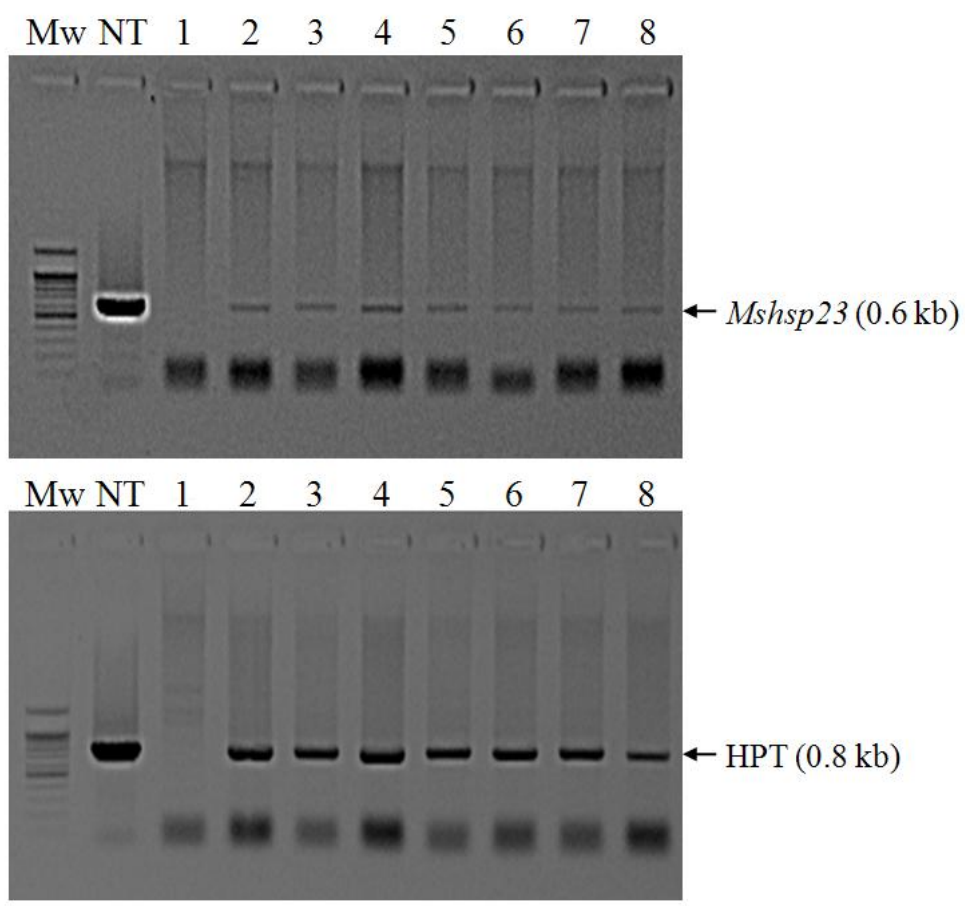

Figure 2. Integration of the transgene into tall fescue plants. PCR amplification of a 630-bp fragment of MsHsp23 (top panel) and an 804-kb fragment of $h p t$ (bottom panel) is shown. 


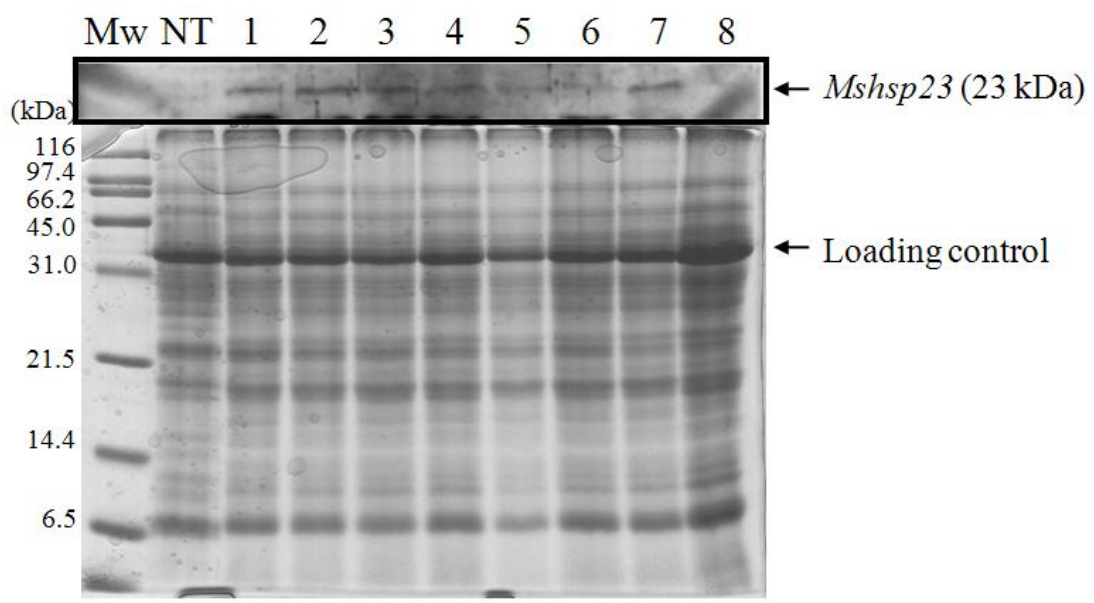

Figure 4. SDS PAGE (top panel) and Western blot analysis of $M s H s p 23$ in transgenic tall fescue leaf protein (bottom panel). Twenty-five micrograms of protein was loaded in each lane, separated on $12.5 \%$ SDS-PAGE, and visualized by CBB staining (upper panel). Mw, marker; NT, non-transgenic control plant; 1-8, transgenic lines.

transgenic and control plants. As shown in Figure 6, transgenic plants accumulate a significantly lower amount of TBARS compared to the controls when exposed to salt and arsenic stresses. However, the accumulation of TBARS in the transgenic plants was similar to that in the control plants under normal conditions. This result is consistent with the ex vitro growth performance of transgenic plants. In addition, our result is in accord with several studies
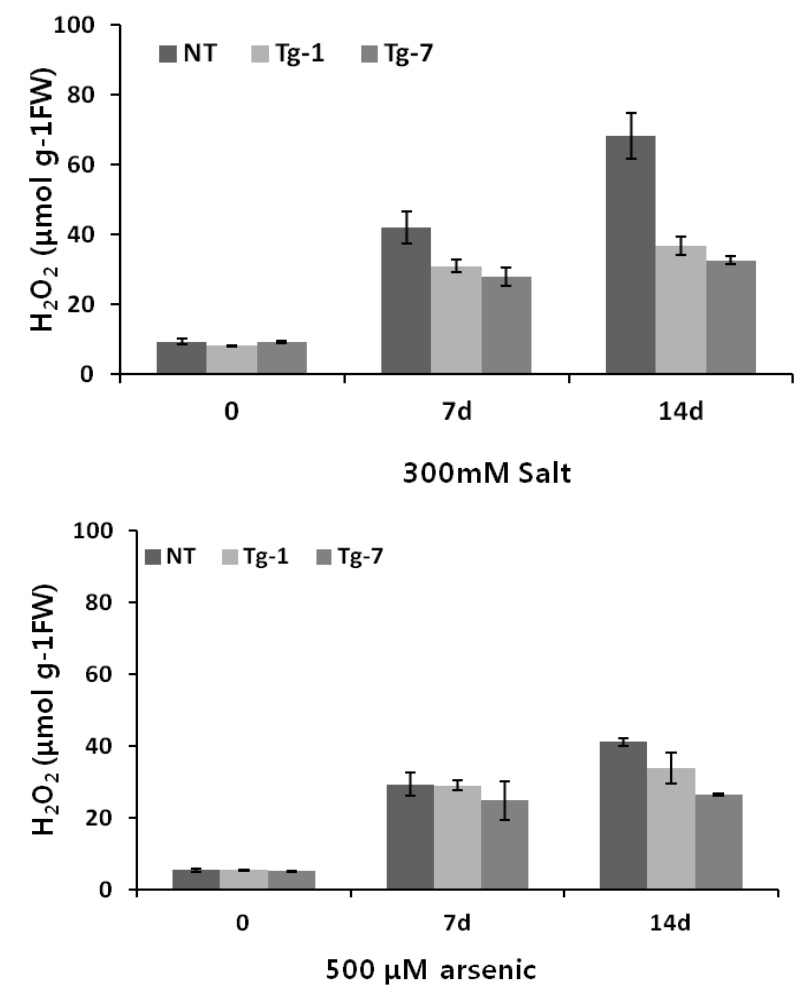

Figure 5. Effects of salt and arsenic stresses on $\mathrm{H}_{2} \mathrm{O}_{2}$ accumulation in leaves of control (NT) and transgenic ( $\mathrm{Tg}-1$, $\mathrm{Tg}-7)$ plants. Data represent the mean and standard deviation (SD) of 3 independent measurements. showing that increased Hsp accumulation can stabilize membrane proteins and result in increased scavenging of ROS under a wide range of stresses including salt and heavy metals (Wang et al., 2003; Ahsan et al., 2007). Reduction in Hsp expression can increase ROS generation and mitochondrial protein oxidation (Yan et al., 2002).

A number of studies have demonstrated the correlation between the accumulation of sHSPs and the acquisition of
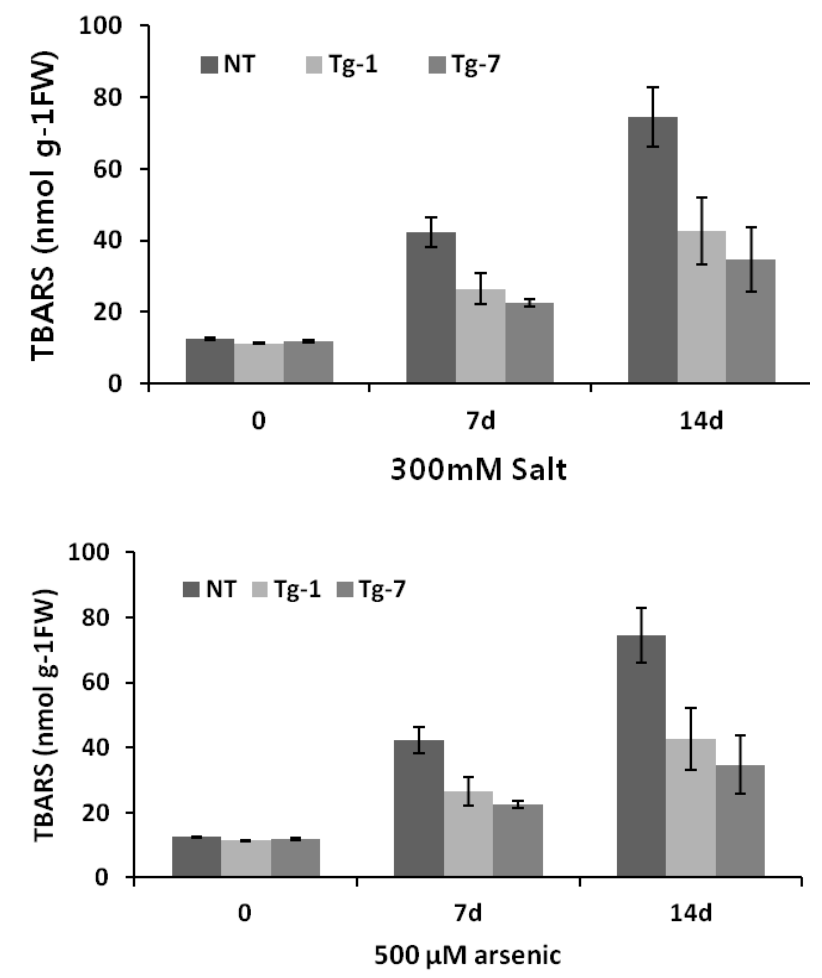

Figure 6. Effects of salt and arsenic stresses on TBARS accumulation in leaves of control (NT) and transgenic (Tg-1, Tg-7) plants. Data represent the means and standard deviation (SD) of 3 independent measurements. 
stress tolerance, including tolerance to heat (Heckathorn et al., 1998; Heckathorn et al., 2002), chilling (Sabehat et al., 1996; Soto et al., 1999; Neta-Sharir et al., 2005), drought (Sato and Yokoya, 2008), and salt (Sugino et al., 1999). However, the in vivo function of plant sHSPs remains largely unknown. A large body of literature suggests that oxidative stress is one of the primary injuries of various abiotic stresses, including salt and heavy metals in plants. The importance of overcoming oxidative stress for tolerance against chilling stress is corroborated by successful examples of the biotechnical engineering of salt and heavy metal tolerance. Overexpression of gamma-ECS conferred significant protection against arsenic toxicity (Dhankher et al., 2002). Alfalfa MsHsp23 gene was cloned and characterized previously (Lee et al., 2012a; Lee et al., 2012b). But there have been no reports on the overexpression of $M s H s p 23$ in forage grasses. To investigate the effect of introducing $M s H s p 23$ in tall fescue, the full-length alfalfa cDNA was introduced into tall fescue plants. The RNA gel blot analysis data showed that MsHsp23 was expressed in transgenic tall fescue plants and that the amount of foreign sHSP was higher. Abiotic stress tolerance of plants is linked not only to chaperon activities but also to the efficiency and stability of the ROSscavenging system (Saruyama and Tanida, 1995).

Therefore, the measurement of TBARS as the product of lipid peroxidation can be used to evaluate tolerance. The lower production of $\mathrm{H}_{2} \mathrm{O}_{2}$ and TBARS in the transgenic tall fescue leaves after salt or arsenic stress may be related to a better ROS-scavenging system. All physiological data suggested that the transgenic tall fescue plants maintained better physiological activity than the non-transgenic plants after salt or arsenic stress. It could be concluded that the salt or arsenic stress tolerance in the transgenic tall fescue is related to the constitutive expression of MsHsp23. Development of new cultivars of tall fescue resistant to various environmental stresses that can be cultivated in such idle lands or the lands with unfavorable environment for cultivation of tall fescue will greatly contribute to the establishment of the production base of high quality forage, improvement of self-sufficiency of foods as well as the development of environment-friendly livestock farming.

\section{ACKNOWLEDGEMENTS}

This study was supported by a grant from the NextGeneration BioGreen 21 Program (No. PJ008139), Rural Development Administration and 2012 Post Doctoral Course Program of National Institute of Animal Science, Rural Development Administration, Republic of Korea.

\section{REFERENCES}

Ahsan, N., D. -G. Lee, S. -H. Lee, K. Y. Kang, J. J. Lee, P. J. Kim,
H. S. Yoon, J. S. Kim and B. -H. Lee. 2007. Excess copper induced physiological and proteomic changes in germinating rice seeds. Chemosphere 67:1182-1193.

Banzet, N., C. Richaud, Y. Deveaux, M. Kazmaier, J. Gagnon and C. Triantaphylides. 1998. Accumulationn of small heat shock proteins, including mitochondrial HSP22, induced by oxidative stress and adaptive response in tomato cells. Plant J. 13:519527.

Cheeseman, J. M. 2007. Hydrogen peroxide and plant stress: A challenging relationship, Plant stress. Global Science Books, pp. 4-15.

Dhankher, O. P., Y. Li, B. P. Rosen, J. Shi, D. Salt, J. F. Senecoff, N. A. Sashti and R. B. Meagher. 2002. Engineering tolerance and hyperaccumulation of arsenic in plants by combining arsenate reductase and gamma-glutamylcysteine synthetase expression. Nat. Biotechnol. 20:1140-1145.

Ekmekci, Y. and S. Terzioglu. 2005. Effects of oxidative stress induced by paraquat on wild and cultivated wheats. Pestic. Biochem. Physiol. 83:69-81.

Eckey-Kaltenbach, H., E. Kiefer, E. Grosskopf, D. Ernst and H. J. Sandermann. 1997. Differential transcript induction of parsley pathogenesis-related proteins and of a small heat shock protein by ozone and heat shock. Plant Mol. Biol. 33:343-350.

Ferullo, J. -M., L. Nespoulous and C. Triantaphylides. 1994. Gamma-ray-induced changes in the synthesis of tomato pericarp protein. Plant Cell Environ. 17:901-911.

Guo, S., W. Wharton, P. Moseley and H. Shi. 2007. Heat shock protein 70 regulates cellular redox status by modulating glutathione related enzyme activities. Cell Stress Chaperones 12:245-254.

Hannaway, D. B., C. Daly, W. Cao, W. Luo, Y. Wei, W. Zhang, A. Xu, C. Lu, X. Shi and X. Li. 2005. Forage species suitability mapping for China using topographic, climatic and soils spatial data and quantitative plant tolerances. Agric. Sci. China. 4:660-667.

Heckathorn, S. A., S. L. Ryan, J. A. Baylis, D. F. Wang, E. W. Hamilton, L. Cundiff and D. S. Luthe. 2002. In vivo evidence from an Agrostis stolonifera selection genotype that chloroplast small heat-shock proteins can protect photosystem during heat stress. Funct. Plant Biol. 29:933-944.

Heckathorn, S. A., C. A. Downs, T. D. Sharkey and J. S. Coleman. 1998. The small, methionine-rich chloroplast heat-shock protein protects photosystem II electron transport during heat stress. Plant Physiol. 116:439-444.

Jiang, Y. and B. Huang. 2000. Effects of drought or heat stress alone and in combination on Kentucky bluegrass. Crop Sci. 40:1358-1362.

Kim, K. -H., I. Alam, K. -W. Lee, S. A. Sharmin, S. -S. Kwak, S. Y. Lee and B. -H. Lee. 2011. Enhanced tolerance of transgenic tall fescue plants overexpressing 2-Cys peroxiredoxin against methyl viologen and heat stresses. Biotechnol. Lett. 32:571576.

Lee, S. -H., N. Ahsan, K. -W. Lee, D. -H. Kim, D. -G. Lee, S. -S. Kwak, S. -Y. Kwon, T. -H. Kim and B. -H. Lee. 2007. Simultaneous overexpression of both $\mathrm{CuZn}$ superoxide dismutase and ascorbate peroxidase in transgenic tall fescue plants confers increased tolerance to a wide range of abiotic stresses. J. Plant Physiol. 164:1626-1638.

Lee, S. -H., D. -G. Lee, H. -S. Woo and B. -H. Lee. 2004. Development of transgenic tall fescue plants from mature 
seed-derived callus via Agrobacterium-mediated transformation. Asian-Aust J. Anim. Sci. 17:1390-1394.

Lee, S. -H., D. -G. Lee, H. -S. Woo, K. -W. Lee, D. -H. Kim, S. -S. Kwak, J. -S. Kim, H. Kim, N. Ahsan, M. S. Choi, J. -K. Yang and B. -H. Lee. 2006. Production of transgenic orchardgrass via Agrobacterium-mediated transformation of seed-derived callus tissues. Plant Sci. 171:408-414.

Lee, K. -W., J. -Y. Cha, K. -H. Kim, Y. -G Kim, B. -H. Lee and S. H. Lee. 2012a. Overexpression of alfalfa mitochondrial HSP23 in prokaryotic and eukaryotic model systems confers enhanced tolerance to salinity and arsenic stress. Biotechnol. Lett. 34: 167-174.

Lee, K. -W., K. -H. Kim, Y. -G Kim, B. -H. Lee and S. -H. Lee. 2012b. Identification of MsHsp23 gene using annealing control primer system. Acta Physiol. Plant. 34:807-811.

Leshem, Y. 1992. Plant membranes: A biophysical approach to structure, development and senescence. Kluwer Academic Publishers: 1-266.

Lin, C. C. and C. H. Kao. 2001. Abscisic acid induced changes in cell wall peroxidase activity and hydrogen peroxide level in roots of rice seedlings. Plant Sci. 160:323-329.

Neta-Sharir, I., T. Isaacson, S. Lurie and D. Weiss. 2005. Dual role for tomato heat shock protein 21: Protecting photosystem II from oxidative stress and promoting color changes during fruit maturation. Plant Cell. 17:1829-1838.
Sato, Y. and S. Yokoya. 2008. Enhanced tolerance to drought stress in transgenic rice plants overexpressing a small heat-shock protein, sHSP17.7. Plant Cell Rep. 27:329-334.

Sugino, M., T. Hibino, Y. Tanaka, N. Nii, T. Takabe and T. Takabe. 1999. Overexpression of DnaK from a halotolerant cyanobacterium Aphanothece halophytica aquires resistance to salt stress in transgenic tobacco plants. Plant Sci. 137:81-88.

Sabehat, A., D. Weiss and S. Lurie. 1996. The correlation between heat shock protein accumulation and persistence and chilling tolerance in tomato fruit. Plant Physiol. 110:531-537.

Soto, A., I. Allona, C. Collada, M. A. Guevara, R. Casado, E. R. Cerezo, C. Aragoncillo and L. Gomez. 1999. Heterologous expression of a plant small heat shock protein enhances Escherichia coli viability under heat and cold stress. Plant Physiol. 120:521-528.

Saruyama, H. and M. Tanida. 1995. Effect of chilling on activated oxygen-scavenging enzymes in low temperature-sensitive and -tolerant cultivars of rice (Oryza sativa L.). Plant Sci. 109: 105-113.

Wang, W., B. Vinocur and A. Altman. 2003. Plant responses to drought, salinity and extreme temperatures: towards genetic engineering for stress tolerance. Planta 218:1-14.

Yan, L. J., E. S. Christians, L. Liu, X. Xiao, R. S. Sohal and I. J. Benjamin. 2002. Mouse heat shock transcription factor 1 deficiency alters cardiac redox homeostasis and increases mitochondrial oxidative damage. EMBO J. 21:5164-5172. 